\title{
Indicadores de movilidad sostenible, análisis y sus perspectivas para el desarrollo en el caso del Cantón Santa Rosa.
}

\author{
Sustainable mobility indicators, analysis and their perspectives for \\ development in the case of the Santa Rosa canton.
}

Joffre Hernán Chica Ambuludí. ${ }^{1}$, Viviana Verónica Carriel Bustamante. ${ }^{2}$ \& Yonimiler Castillo Ortega. ${ }^{3}$

Recibido: 11-12-2020 / Revisado: 03-01-2021 /Aceptado: 11-01-202021/ Publicado: 05-02-2021

\begin{abstract}
:
DOI: $\underline{\text { https://doi.org/10.33262/concienciadigital.v4i1.1.1544 }}$

Introduction. Sustainable mobility constitutes a national and local development strategy, framed in the Sustainable Development Goals agreed by UN member countries and consists of promoting and prioritizing less polluting means and modes of transport that allow satisfying the mobility needs of the population on equitable terms. Objective. Propose a base set of indicators that measure cantonal mobility with a sustainability approach, that constitute tools for decision-making by local authorities and that finally allow improving the standard of living of users and the general population; with accessibility, adequate service levels and protection of the environment. Methodology. The research design was quantitative, descriptive and analytical. The Origin / Destination survey was taken in October 2020 and it was applied in 428 homes distributed in the Canton Santa Rosa, it was consulted about the characteristics of the trips of each of the residents of the home, the number of residents of whom collected information is 1,364. Results. The main results show that the total number of daily trips in the Santa Rosa Canton is 76,327 in all modalities, by all means and for

\footnotetext{
${ }^{1}$ Universidad Católica de Cuenca, Posgrado, Maestría en Desarrollo Local Mención en Ordenamiento Territorial, Cuenca, Ecuador, joffre.chica@est.ucacue.edu.ec, https://orcid.org/0000-0002-2295-7378

${ }^{2}$ Universidad Católica de Cuenca, Carrera de economía), Cuenca, Ecuador, viviana.carriel@ucacue.edu.ec https://orcid.org/0000-0001-6572-0648

${ }^{3}$ Universidad Católica de Cuenca, Posgrado, Maestría en Desarrollo Local Mención en Ordenamiento Territorial, Cuenca, Ecuador, ycastilloo@ucacue.edu.ec, https://orcid.org/0000-0002-7710-5199
} 
different reasons of travel; the average IPK in bus operation in intracantonal transport is 5.1; the average distance traveled by public transport is $2.6 \mathrm{~km}(63.06 \%)$, by private car $4.7 \mathrm{~km}$ (48.36\%), on foot $0.7 \mathrm{~km}(97.53 \%)$; the modal split is as follows: urban bus $21.04 \%$, own vehicle $16.72 \%$, intra or interprovincial bus $10.75 \%$, motorcycle $9.89 \%$, passenger in own vehicle $9.73 \%$, conventional taxi $6.91 \%$, on foot $6.36 \%$, bicycle $5.10 \%$, trike $3.77 \%$, informal taxi $3.53 \%$, truck or tipper $2.28 \%$, double cab van $1.33 \%$, Executive taxi $1.18 \%$, single cab van $0.71 \%$, informal van $0.55 \%$, and school or institutional $0.16 \%$; The main reasons for travel are to work with $43.1 \%$, purchases $15.7 \%$ and paperwork $10.8 \%$. It is concluded that $21.4 \%$ of the trips are made by intracantonal public transport and $11.46 \%$ are made on foot or by bicycle, values that indicate a low inclination to sustainable modes of transport.

Keywords: Indicators of sustainable mobility, transport, public transport, transit, roads.

\section{Resumen.}

Introducción. La movilidad sostenible constituye una estrategia de desarrollo nacional y local, enmarcada en los Objetivos de Desarrollo Sostenible acordados por países miembros de la ONU y consiste en promover y priorizar medios y modos de transporte menos contaminantes y que permitan satisfacer las necesidades de movilidad de la población en términos equitativos. Objetivo. Proponer un conjunto base de indicadores que midan la movilidad cantonal con enfoque de sostenibilidad, que constituya herramientas para la toma de decisiones de autoridades locales y que finalmente permita mejorar el nivel de vida de usuarios y de la población en general; con accesibilidad, niveles de servicio adecuados y protección del medio ambiente. Metodología. El diseño de investigación fue cuantitativa, de tipo descriptiva y analítica. Se procedió a tomar la encuesta Origen/Destino en octubre de 2020 y se aplicaron en 428 hogares repartidos en el Cantón Santa Rosa, se consultó acerca de características de los viajes de cada uno de los residentes del hogar, el número de residentes de quienes se recabó información es de 1.364. Resultados. Los principales resultados muestran que el número total de viajes diarios en el Cantón Santa Rosa es de 76.327 en todas las modalidades, por todos los medios y por los diferentes motivos de viaje; el IPK promedio en operación de buses en transporte intracantonal es de 5.1; la distancia promedio viajes en transporte público es de 2,6 km (63,06\%), en auto particular 4,7 km $(48,36 \%)$, a pie $0,7 \mathrm{~km}(97,53 \%)$; la partición modal es como sigue: bus urbano $21,04 \%$, vehículo propio $16,72 \%$, bus intra o interprovincial $10,75 \%$, motocicleta $9,89 \%$, acompañante en vehículo propio $9,73 \%$, taxi convencional $6,91 \%$, a pie $6,36 \%$, bicicleta $5,10 \%$, tricimoto $3,77 \%$, taxi informal $3,53 \%$, camión o volquete $2,28 \%$, camioneta doble cabina $1,33 \%$, taxi Ejecutivo 1,18\%, camioneta cabina simple 0,71\%, camioneta informal $0,55 \%$, y escolar o institucional $0,16 \%$; los principales motivos de viaje son al Trabajo con $43,1 \%$, compras $15,7 \%$ y , trámites $10,8 \%$. Se concluye que el $21.4 \%$ de los viajes se realizan en transporte público intracantonal y el $11.46 \%$ se realizan a pie o en bicicleta, valores que indican una baja inclinación a modos de transporte sostenible. 
Palabras clave: Indicadores de movilidad sostenible, transporte, transporte público, tránsito, vialidad.

\section{Introducción.}

La movilidad se ha constituido en uno de los principales temas de discusión al momento de abordar los objetivos de desarrollo de las urbes en general. La movilidad y especialmente el transporte tienen la capacidad de modificar el patrón de funcionamiento de una ciudad o región. Mediante la aplicación de política pública específica es posible lograr mejores condiciones de equidad, accesibilidad y ofrecer oportunidades a la población que no está integrada adecuadamente por las limitaciones físicas o de capacidad adquisitiva.

El crecimiento poblacional en Latinoamérica crea necesidades de diversa índole que incluye las inversiones en infraestructura para la dotación de los diferentes servicios y entre ellos el transporte. (Ortiz, Herrera y Sánchez 2003)

Entre los servicios importantes que permite el adecuado funcionamiento de los centros poblados está el transporte, englobado en el concepto de movilidad, que tiene la capacidad de influir positivamente en la calidad de vida de los habitantes.

La movilidad es de interés general, de ahí la importancia de este estudio. Según Eguiguren desde mediados del siglo anterior contamos con un modelo centrado en el uso del auto privado, caracterizado por la contaminación, alta utilización de energía, mal uso del espacio físico y ahora la congestión. (EGUIGUREN, 2015) por lo que es de suma importancia analizar el problema del transporte público en general, mediante indicadores de movilidad sostenible con propuestas para el desarrollo local.

Esto es particularmente relevante puesto que la movilidad sostenible, según definición del Consejo de Transportes de la UE, permitirá dar respuesta a las necesidades básicas de acceso y desarrollo de la población, a la vez fomentará la igualdad intergeneracional; resulta asequible, opera con equidad y con eficacia. (IDAE, 2006). (Instituto para la Diversificación y Ahorro 2006)

\section{Breve descripción del Cantón Santa Rosa.}

El Cantón Santa Rosa se ubica geográficamente en la provincia de El Oro, en la región costa de Ecuador. Su cabecera cantonal es la ciudad de Santa Rosa, lugar donde se agrupa gran parte de sus habitantes. Su población para el año 2010 era de 69.036 habitantes según el censo de ese año y según proyecciones del INEC para 2020 Santa Rosa contaría con alrededor de 80.000 habitantes. Tiene una superficie de $889 \mathrm{~km} 2$, con un parque automotor de 10.000 vehículos aproximadamente. 


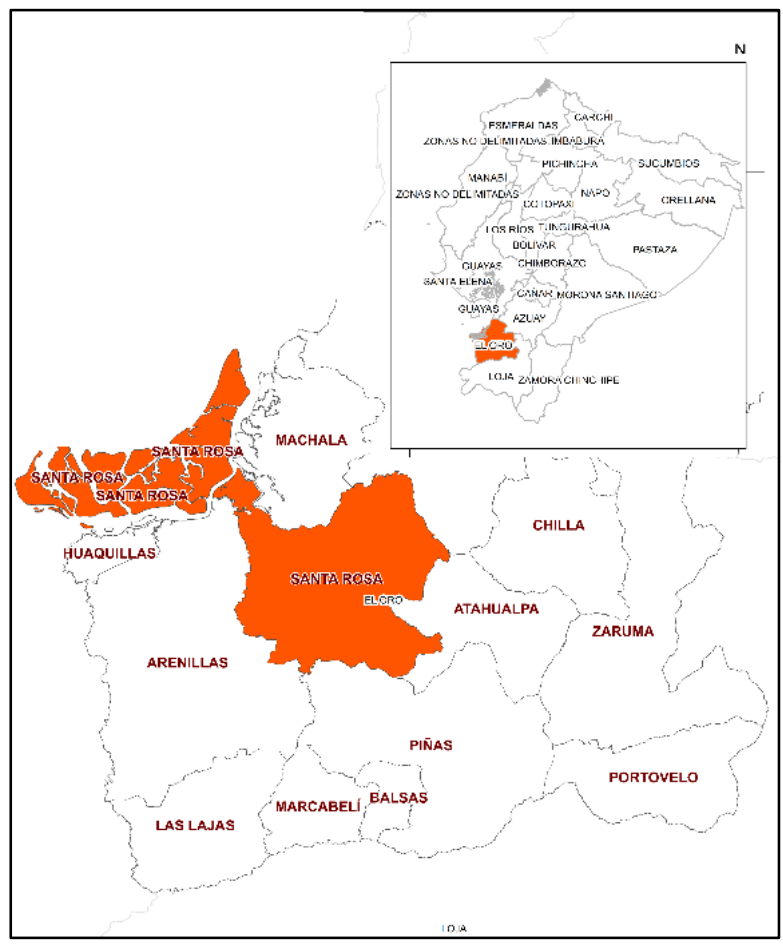

Gráfico. 1. Ubicación del cantón Santa Rosa. Elaborado por el autor.

Santa Rosa está asentada en una región rica y productiva del país en donde destacan la producción agrícola, acuícola y minera, tiene una considerable extensión territorial, un gran desarrollo económico y social por lo que existe un constante movimiento de sus habitantes dentro y hacia el casco urbano.

El cantón está formado por 8 parroquias: Santa Rosa, Bellavista, La Avanzada, La Victoria, San Antonio, Torata, Bellamaría y Jambelí. (GAD MUNICIPAL DEL CANTÓN SANTA ROSA, 2020)

Santa Rosa en general tiene buena conectividad vial regional por medio de la red de vías troncales nacionales, su territorio está atravesado por la vía troncal E25 que permite la conectividad al norte con Machala, la capital provincial, y al noreste con la región Sierra, al sur permite la conectividad con los cantones vecinos Arenillas y el limítrofe Cantón uaquillas y al sur este permite la conexión con la parte alta de la provincia y el sur de la Sierra ecuatoriana. La vía 584A permite la conectividad con el Cantón Pasaje y también la parte alta de la provincia. En tanto que la vía estatal No. 586 permite trasladarse a Puerto Pitahaya.

La vialidad de Santa Rosa tanto en la parte urbana como rural se puede decir que está en buen estado, sin embargo existen problemas de movilidad en la parte urbana relacionados con el parqueo vehicular, circulación peatonal, congestión, problemas de inseguridad vial y un sistema de transporte público que si bien da cobertura a gran parte del cantón y permite a la población movilizarse en tiempos y condiciones relativamente buenas, requiere modernizarse operacionalmente y mejorar sus prestaciones.

Para el ejercicio de la competencia de planificar, gestionar regular y controlar el transporte, tránsito y seguridad vial en el cantón se creó la Empresa Pública Municipal de Transito Transporte Terrestre Fluvial Marítimo Seguridad Vial Terminal Terrestre y Movilidad de Santa Rosa - EMOVTT SR EP, que tiene por objetivo principal asegurar una eficiente movilidad dentro del cantón.

Al momento de movilizarse dentro del cantón las personas privilegian el uso de vehículo particular y comercial (taxis y camionetas) y no prefieren medios alternos como caminata o 
el uso de bicicleta o el uso de transporte público intracantonal, lo que ocasiona congestión, contaminación, inseguridad y problemas serios al momento de desplazarse.

En el cantón Santa Rosa, el actual sistema de movilidad no es eficiente, no se prioriza el transporte público sobre el individual, no se promueve el transporte no motorizado y los

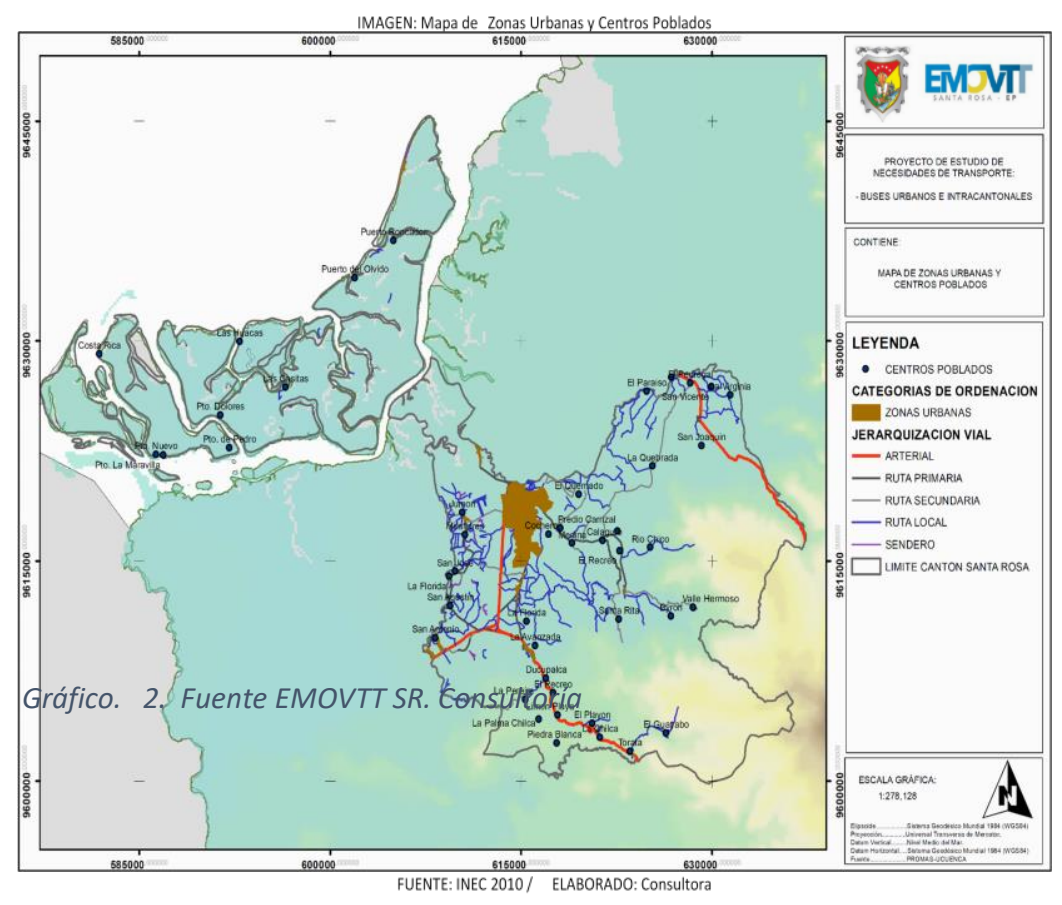

viajes a pie, por lo que el presente proyecto propone fomentar la movilidad sostenible y transporte público integral, sustentable, seguro, eficiente, para el desarrollo local.

Lo que se busca en esta investigación es realizar el análisis de las condiciones de movilidad local, desde la perspectiva de la movilidad sostenible y proponer indicadores específicos que permitan medir en un

horizonte de mediano y largo plazo la evolución de las condiciones de movilidad en el Cantón.

\section{Marco Teórico.}

\section{Movilidad Sostenible.}

Carolina Álvarez en el documento "La movilidad sostenible como política global y su consolidación hacia el futuro en la ciudad de Medellín en respuesta a la ocupación territorial y sus problemáticas" en relación a la Movilidad Sostenible dice: "una manera simple de definir movilidad sostenible, es entendiéndola como un sistema de movilidad que integra sus distintos modos de transporte, que da respuesta a las necesidades actuales y futuras de movilidad y que utiliza energías limpias y/o renovables. De ahí que sean elementos inseparables de una movilidad sostenible: la reducción de emisiones contaminantes producidas por fuentes móviles, el uso de combustibles limpios y nuevas tecnologías para el transporte, la construcción de redes peatonales y cicleables y la promoción de su uso, la optimización de sistemas de transporte masivos alrededor de un ordenamiento territorial que sea coherente con estas iniciativas. En este sentido, la relación entre el uso del suelo y la 
movilidad debe ser indisoluble puesto que los problemas asociados a la movilidad son multicausales y esta es transversal a las prácticas urbanas". (Álvarez, 2016)

Por otra parte, luego de una época caracterizada por buscar el crecimiento económico a toda costa y el desarrollismo aparece la sostenibilidad y movilidad sostenible, cuando se enfatizó la defensa del medio ambiente frente al crecimiento económico que no toma en cuenta los efectos nocivos sobre éste. (Flores Juca, y otros 2017)

La movilidad sostenible entonces promueve que las autoridades nacionales y locales traten de construir modelos de transporte eficientes, que mejoren la accesibilidad de todos los grupos sociales, tanto accesibilidad física como accesibilidad económica, con la finalidad de hacer también viajes más seguros y por último incrementar o mejorar la calidad de vida de la población.

“...la movilidad se expresa en el transporte. Es decir, la movilidad es un concepto más amplio que el de transporte y el de transporte público colectivo. Este último comprende aquellos medios que permiten el traslado masivo de personas de un lugar a otro dentro de la ciudad, y que son regulados u operados por un organismo estatal; se considera público desde la perspectiva jurídica por ser un servicio de interés para la sociedad en general, independientemente de quién realice su prestación.” (García-Schilardi 2013)

\section{Evolución del concepto Movilidad Sostenible}

Holden, en cambio dice que la aplicación del imperativo del desarrollo sostenible al sector del transporte ha dado lugar a varios conceptos que se denotan con términos como: movilidad sostenible, transporte sostenible, transporte sostenible y sistemas de transporte sostenibles. En la literatura sobre transporte y desarrollo sostenible estos términos son esencialmente sinónimos. El término "transporte sostenible" parece ser el preferido en Norteamérica, mientras que "movilidad sostenible" se prefiere en Europa. Sin embargo, según Tengström, citado por Holden, debería hacerse una distinción clara entre un sistema de transporte sostenible y movilidad sostenible, porque esforzarse por lograr una movilidad sostenible es muy diferente de esforzarse por lograr un sistema de transporte sostenible. Sin embargo, consideró que es más fructífero centrarse en el contenido de los conceptos que en los términos utilizados para designarlos. (Holden 2007)

A mediados del siglo anterior las disciplinas relacionadas con el transporte y el tránsito y asociada a esta última la seguridad vial, generalmente se las estudiaba por separado. La Planificación del Transporte estaba fuertemente condicionada por el desarrollo de la vialidad e infraestructura, así también el tránsito estuvo muy asociado a las facilidades para los vehículos, en las medidas para incrementar la capacidad vial y reducir la congestión.

Holden identifica la evolución del concepto movilidad sostenible cronológicamente en tres períodos: 
Primero. (1992-1993). - Cuando el objetivo parecía centrarse en ver la forma de reducir la duración y número de viajes motorizados. En este periodo la Comisión Europea acuñó el término movilidad sostenible.

Segundo. Los años intermedios (1993-2000). - A más de Europa, en este período, varias instituciones supranacionales comienzan a difundir el concepto de movilidad sostenible como el Banco Mundial.

Tercero. Los años posteriores (2000-2005). - En este período se reconoce con mayor fuerza que lograr una movilidad sostenible requiere algo más que un conocimiento mejorado sobre soluciones tecnológicas.

Pozueta, 2000, hace una síntesis de las teorías y sustento teórico de la movilidad sostenible y que se presenta a continuación: Existe la discusión entre densificación de la trama urbana o la dispersión. En muchos casos se urbaniza con características de baja densidad, este tipo de configuración de las urbes promueve el mayor uso del auto particular ante la dificultad de proveer de otros medios de transporte que en estas circunstancias resulta costosa su operación. (Pozueta 2000).

Existe actualmente la discusión entre ciudades compactas y dispersas. Adoptar la política de ciudades compactas, en las actuales circunstancias, amerita analizar la planificación integral de las ciudades y su relación con un sistema de transporte óptimo y equitativo.

\section{Movilidad Sostenible y desarrollo local.}

La Asamblea Constituyente de 2008 determinó que sean los Municipios del Ecuador los encargados de planificar, regular y controlar el transporte, tránsito y seguridad vial. En el año 2008 se promulga la Ley Orgánica de Transporte terrestre Tránsito y Seguridad Vial la que introduce conceptos antes no contemplados anteriormente como: el derecho a la vida, al libre tránsito y la movilidad, calidad de vida del ciudadano, preservación del ambiente, desconcentración y descentralización interculturalidad e inclusión a personas con discapacidad.

El nuevo esquema de competencias establece que sean los GADs los que tomen las competencias citadas, oficialmente mediante la Resolución No.006-CNC-2012 (Consejo Nacional de Competencias 2012).

\section{Movilidad Sostenible y Selección de Indicadores.}

Se considera generalmente que para diagnosticar una situación base hay que hacer evaluaciones y luego de la implementación de mejoras se deben hacer mediciones y es donde cobra importancia el sistema de indicadores y específicamente los de movilidad sostenible. El análisis conjunto de los indicadores nos aproxima a realizar una valoración general de los patrones de movilidad y transporte público en términos del grado de sostenibilidad, y también 
a reconocer diferencias según Zonas de Transporte. Así, las áreas centrales de la ciudad, compactas y diversas funcionalmente, presentan en general unos patrones de movilidad más sostenible. (Salado, 2006). (Díaz Muñoz, y otros 2007)

Por ser la movilidad un fenómeno económico y social, para la creación de un sistema de indicadores se recomienda tener: Indicadores económicos, sociales, ambientales e indicadores específicos (transporte, tránsito y seguridad vial), la presente investigación se centró en los últimos.

\section{Experiencia en ciudades latinoamericanas.}

Un estudio de la Corporación Andina de Fomento hace seguimiento al sistema de movilidad de ciudades representativas de Latinoamérica. Donde el transporte colectivo predomina en 9 de las 15 ciudades. (CAF 2011) De este estudio se han tomado varios indicadores que se proponen para el caso de Santa Rosa.

La movilidad promedio en las ciudades estudiadas es de dos viajes diarios por habitante. Los niveles más altos de utilización de transporte colectivo por habitante corresponden a México, Santiago y Lima.

En nuestro país el avance en temas de movilidad sostenible es parcial y no existe bibliografía abundante. En el año 2014 por parte del Consejo Nacional de Competencias se emitió una Guía Metodológica para elaboración de Planes de Movilidad y en la cual se toman en cuenta consideraciones de movilidad sostenible (Núñez, 2014). (Consejo Nacional de Competencias. 2014).

Con la emergencia por la pandemia mundial algunas condiciones han cambiado, tal es el caso de la ocupación de las unidades de transporte público y comercial, pues por temas de distanciamiento social se trata de reducir los viajes y aglomeraciones. A la vez esta reducción de la demanda de transporte público y comercial puede ser una oportunidad para fomentar lo que se ha llamado modos de transporte alternativos como la bicicleta o caminar que pueden colaborar con el distanciamiento físico recomendado.

\section{Indicadores de movilidad sostenible propuestos para Santa Rosa.}

Escoger los indicadores que se analizarán para Santa Rosa, supuso el análisis de la literatura nacional y extranjera existente. Las metodologías internacionales sugieren agruparlos por categorías, por ejemplo, transporte, salud, economía, etc. (Motos 2019)

En Ecuador la adopción de indicadores de movilidad y específicamente de movilidad sostenible en los GADs es parcial y poco consistente ya que dependen de el criterio del consultor contratado para desarrollar los PMS y de los requerimientos del contratante.

En el año de 2012 el MTOP, ente rector de la movilidad en Ecuador, publicó el documento "Herramientas de gestión para el transporte terrestre"., en el que se recomienda, en relación 
ISSN: 2600-5859

Vol. 4, N¹.1, p. 22-43, Febrero, 2021

al desarrollo de estudios y planes de movilidad: "Los indicadores que se definan se evaluarán anualmente, lo que permitirá ver cómo se van cumpliendo los objetivos del plan. Estos indicadores deberán responder a las normas o a los objetivos establecidos por las autoridades nacionales en cuanto a movilidad, medio ambiente y accesibilidad". (Ministerio de Transporte y Obras Públicas. 2012)

Posteriormente en el año 2014 por parte del Consejo Nacional de Competencias se emitió una Guía Metodológica para elaboración de Planes de Movilidad y en la cual se toman en cuenta consideraciones de movilidad sostenible. Se indica que la objetividad y la claridad de los indicadores de las variables analizadas facilitarán la coordinación y la participación del resto de actores involucrados. El estudio recomienda agrupar los indicadores por componentes.

El documento Identificación y análisis de indicadores de sostenibilidad para la movilidad, publicado en la Revista de la Facultad de Arquitectura y Urbanismo de la Universidad de Cuenca también en un referente importante en la definición de propuesta de indicadores.

A partir de la revisión de las experiencias en ciudades de Latinoamérica, de la literatura internacional, de las herramientas publicadas a nivel nacional y de consultas con especialistas en el tema, a continuación se proponen los indicadores a ser evaluados tomando como criterio lo viable en función de la información que generalmente se dispone en los elementos de planificación territorial y sectorial que dispone el Municipio de Santa Rosa y en función de la capacidad de obtener información de fuente primaria mediante encuestas. A continuación, los indicadores propuestos:

Tabla 1. Indicadores de movilidad sostenible y fórmulas de cálculo.

\begin{tabular}{|c|c|c|}
\hline $\begin{array}{c}\text { Código } \\
\text { de } \\
\text { indicador }\end{array}$ & Indicador de Transporte & Fórmula de cálculo \\
\hline TP1 & Flota de transporte público & $\begin{array}{l}\text { Suma de las capacidades individuales / Número de unidades de } \\
\text { Transporte público intracantonal }\end{array}$ \\
\hline TP2 & $\begin{array}{l}\text { Flota de transporte } \\
\text { comercial }\end{array}$ & $\begin{array}{l}\text { Número de unidades por cada modalidad de transporte comercial } \\
\text { / Población en miles Cantón Santa Rosa. }\end{array}$ \\
\hline TP3 & Empleo generado & $\begin{array}{l}\text { Número de empleos directos en operadoras de transporte / PEA } \\
\text { cantonal. }\end{array}$ \\
\hline TP4 & IPK (pasajeros por km) & $\begin{array}{l}\text { Promedio de el número de pasajeros transportados en transporte } \\
\text { público / Número de kilómetros recorridos }\end{array}$ \\
\hline TP5 & $\begin{array}{l}\text { PVD } \\
\text { (pasajeros/vehículo/día) en } \\
\text { transporte público y taxi }\end{array}$ & $\begin{array}{l}\text { Número de pasajeros transportados / Número de unidades } \\
\text { laborando }\end{array}$ \\
\hline TP6 & $\begin{array}{l}\text { RMD (km/vehículo/día) en } \\
\text { transporte público. }\end{array}$ & $\begin{array}{l}\text { Número de km recorridos por flota de buses / Número de } \\
\text { unidades de transporte público }\end{array}$ \\
\hline TP7 & $\begin{array}{l}\text { Gasto promedio usuario de } \\
\text { transporte público por } \\
\text { motivo estudios. }\end{array}$ & $\begin{array}{l}\text { Gasto total en transporte por motivo estudios / Número de viajes } \\
\text { por motivos estudios }\end{array}$ \\
\hline TP8 & $\begin{array}{l}\text { Gasto promedio usuario de } \\
\text { transporte público por } \\
\text { motivo trabajo }\end{array}$ & $\begin{array}{l}\text { Gasto total en transporte por motivo trabajo / Número de viajes } \\
\text { por motivos de trabajo }\end{array}$ \\
\hline
\end{tabular}




\begin{tabular}{|c|c|c|}
\hline TP9 & $\begin{array}{l}\text { Gastos en transportación en } \\
\text { función del nivel de renta } \\
\text { familiar disponible }\end{array}$ & $\begin{array}{l}\text { (Ingreso mínimo por número de perceptores) X \% de gasto } \\
\text { como índice de INEC }\end{array}$ \\
\hline TP10 & $\begin{array}{l}\text { Uso de transporte público } \\
\text { colectivo }\end{array}$ & $\begin{array}{l}\text { (Número de viajes en transporte público / Número de viajes } \\
\text { totales en el cantón) X } 100\end{array}$ \\
\hline TP11 & $\begin{array}{l}\text { \% de viajes por ocupación- } \\
\text { empleo. }\end{array}$ & $\begin{array}{l}\text { (Número de viajes por cada ocupación y empleo / Número total } \\
\text { de viajes en el cantón) X } 100\end{array}$ \\
\hline TP12 & $\%$ de viajes por escolaridad & $\begin{array}{l}\text { (Número de viajes por cada nivel de escolaridad / Número total } \\
\text { de viajes en el cantón) X } 100\end{array}$ \\
\hline TP13 & $\begin{array}{l}\text { Distancia de los viajes en } \\
\text { transporte público }\end{array}$ & $\begin{array}{l}\text { (Sumatoria de las distancias de viajes en transporte público / } \\
\text { Número de viajes en transporte público) }\end{array}$ \\
\hline TP14 & $\begin{array}{l}\text { Distancia de los viajes en } \\
\text { auto particular }\end{array}$ & $\begin{array}{l}\text { (Sumatoria de las distancias de viajes en transporte particular / } \\
\text { Número de viajes en transporte particular) }\end{array}$ \\
\hline TP15 & Distancia de los viajes a pie & $\begin{array}{l}\text { (Sumatoria de las distancias de viajes a pie / Número de viajes a } \\
\text { pie) }\end{array}$ \\
\hline TP16 & $\begin{array}{l}\text { Distancia de los viajes en } \\
\text { bicicleta }\end{array}$ & $\begin{array}{l}\text { (Sumatoria de las distancias de viajes en bicicleta / Número de } \\
\text { viajes en bicicleta) }\end{array}$ \\
\hline TP17 & Partición modal & $\begin{array}{l}\text { (Número de viajes por modalidad / número total de viajes) } \mathrm{X} \\
100\end{array}$ \\
\hline TP18 & Motivo de viaje & $\begin{array}{l}\text { (Número de viajes por motivo / número de total de viajes) X } \\
100\end{array}$ \\
\hline TP19 & $\begin{array}{l}\text { Tiempo de los viajes en } \\
\text { transporte público }\end{array}$ & $\begin{array}{l}\text { (Sumatoria de tiempos de viaje en transporte público / Número } \\
\text { de viajes en transporte público) }\end{array}$ \\
\hline TP20 & $\begin{array}{l}\text { Tiempo de los viajes en auto } \\
\text { particular }\end{array}$ & $\begin{array}{l}\text { (Sumatoria de tiempos de viaje en transporte particular / Número } \\
\text { de viajes en transporte particular) }\end{array}$ \\
\hline TP21 & Tiempo de los viajes a pie & (Sumatoria de tiempos de viaje a pie / Número de viajes a pie) \\
\hline TP22 & $\begin{array}{l}\text { Tiempo de los viajes en } \\
\text { bicicleta }\end{array}$ & $\begin{array}{l}\text { (Sumatoria de tiempos en bicicleta / Número de viajes en } \\
\text { bicicleta) }\end{array}$ \\
\hline TP23 & $\begin{array}{l}\text { Capacidad vehicular del } \\
\text { transporte público colectivo } \\
\text { por viajes y por habitantes. }\end{array}$ & $\begin{array}{l}\text { (Capacidad del transporte público colectivo / número de viajes } \\
\text { total) X 100. (Capacidad del transporte público colectivo / } \\
\text { Número de habitantes) X } 100\end{array}$ \\
\hline ТP24 & $\begin{array}{l}\text { Velocidad promedio de } \\
\text { circulación transporte } \\
\text { público. }\end{array}$ & $\begin{array}{l}\text { Distancia promedio recorrida / Tiempo promedio empleado para } \\
\text { el desplazamiento }\end{array}$ \\
\hline TP25 & $\begin{array}{l}\text { Edad del parque automotor } \\
\text { del transporte público } \\
\text { colectivo }\end{array}$ & $\begin{array}{l}\text { (Sumatoria de la edad de las unidades que prestan servicio de } \\
\text { transporte público colectivo / Total de unidades) }\end{array}$ \\
\hline $\begin{array}{c}\text { Código } \\
\text { de } \\
\text { indicador }\end{array}$ & Indicador de Tránsito & Fórmula de cálculo \\
\hline TR1 & Índice de motorización & $\begin{array}{l}\text { [ Número de automotores registrados anualmente en el cantón / } \\
\text { Número de habitantes en el cantón] X } 1000\end{array}$ \\
\hline TR2 & $\begin{array}{l}\text { Viajes en medios no } \\
\text { motorizados }\end{array}$ & $\begin{array}{l}\text { (Suma de Viajes realizados a pie y en bicicleta / Total de Viajes } \\
\text { realizados según las encuestas realizadas) X } 100\end{array}$ \\
\hline TR3 & $\begin{array}{l}\text { Accidentabilidad de tránsito } \\
\text { anual }\end{array}$ & $\begin{array}{l}\text { (Número total anual de siniestros, heridos y muertos / Población } \\
\text { total del área de estudio) X } 100.000\end{array}$ \\
\hline TR4 & $\begin{array}{l}\text { Tenencia de vehículos en } \\
\text { hogares. }\end{array}$ & (Número de vehículos por clase / Número de hogares) \\
\hline TR5 & $\begin{array}{l}\text { Vehículos que consumen } \\
\text { energía alternativa o } \\
\text { renovable }\end{array}$ & $\begin{array}{l}\text { = (Número de vehículos matriculados en el cantón Santa Rosa } \\
\text { que utilizan energía alternativa - renovable / Número de } \\
\text { vehículos matriculados en el cantón Santa Rosa) X } 100\end{array}$ \\
\hline
\end{tabular}




\begin{tabular}{|c|c|c|}
\hline $\begin{array}{l}\text { Código } \\
\text { de } \\
\text { indicador }\end{array}$ & Indicador de vialidad & Fórmula de cálculo \\
\hline V1 & Km de vía & $\begin{array}{l}\text { Número de km totales por cada tipo de vía. Porcentaje por cada } \\
\text { tipo de vía }\end{array}$ \\
\hline V2 & $\begin{array}{l}\text { Intersecciones } \\
\text { semaforizadas }\end{array}$ & $\begin{array}{l}\text { (Número de intersecciones semaforizadas / Número de } \\
\text { intersecciones) X } 100\end{array}$ \\
\hline V3 & $\begin{array}{l}\text { Vías con prioridad para } \\
\text { peatones }\end{array}$ & $\begin{array}{l}\text { (Kilómetros de vías con prioridad para peatones / Número de km } \\
\text { de vías de hormigón o asfalto) X } 100\end{array}$ \\
\hline V4 & $\begin{array}{l}\text { Vías con prioridad para } \\
\text { ciclistas }\end{array}$ & $\begin{array}{l}\text { (Kilómetros de vías con prioridad para bicicletas / Número de } \\
\text { km de vías de hormigón o asfalto) X } 100\end{array}$ \\
\hline V5 & $\begin{array}{l}\text { Acceso a personas con } \\
\text { movilidad especial en la } \\
\text { parada }\end{array}$ & $\begin{array}{l}\text { (Número de paradas con facilidades para personas con } \\
\text { discapacidad / Número total de paradas de bus) X } 100 \text {. } \\
\text { (Número de paradas con rampas de acceso / Número total de } \\
\text { paradas de bus) X } 100 \text {. }\end{array}$ \\
\hline V6 & Puntos seguros de paradas & $\begin{array}{l}\text { (Número de paradas con infraestructura / Número total de } \\
\text { paradas) X } 100\end{array}$ \\
\hline V7 & $\begin{array}{l}\text { Velocidad de } \\
\text { desplazamiento media del } \\
\text { transporte público. }\end{array}$ & $\begin{array}{l}\text { (Distancia promedio de recorrido / Tiempo promedio de } \\
\text { transporte público. }\end{array}$ \\
\hline V8 & $\begin{array}{l}\mathrm{km} \text { del sistema del } \\
\text { transporte por } 100,000 \\
\text { habitantes }\end{array}$ & $\begin{array}{l}\text { (Número de kilómetros de sistema de transporte público / } 100 \\
\text { mil habitantes) }\end{array}$ \\
\hline
\end{tabular}

Elaborado por el autor

\section{Levantamiento de la Información.}

La mayor parte de indicadores propuestos se basan en la encuesta levantada y en fuente secundaria como por ejemplo el Plan de Ordenamiento Territorial de Santa Rosa, El Plan de Movilidad de Santa Rosa, el Estudio de Oferta y Demanda de transporte en el Cantón Santa Rosa y el estudio de Costos de Operación de transporte público y comercial.

La investigación e información de campo corresponde al Cantón Santa Rosa que para el año 2020, según el INEC cuenta con una población de 82.171 habitantes distribuida en las 8 parroquias, pero el $77 \%$ de la misma se encuentra en la cabecera cantonal.

Como herramienta de campo principal se utilizó la encuesta Origen/Destino, que es un método descriptivo con el que se pueden detectar ideas, necesidades, preferencias, hábitos de uso del transporte, etc. La característica de las encuestas O-D es que por medio de estas se obtiene información de número de viajes, miembros de familia, posesión de vehículos, número de viajes, modalidad, etc. La encuesta O/D se utiliza ampliamente con la finalidad de conocer los patrones de movilidad de un territorio determinado. (Villa, 2016).

Se procedió a tomar la encuesta Origen/Destino en octubre de 2020 y se aplicaron en 428 hogares repartidos en el Cantón Santa Rosa, en la encuesta se consultó acerca de los viajes realizados el día anterior por cada uno de los residentes del hogar. El número de residentes de quienes se recabó información es de 1.364. El número total de viajes detectados en las encuestas fue de 1.267 en todas las modalidades, en todos los medios y por los diferentes motivos de viaje. Los viajes encontrados en la muestra se expanden al universo que es la población total del cantón Santa Rosa proyectado al año 2020 y se obtiene una estimación 
total de viajes diarios para el Cantón de 76.327 en todas las modalidades, por todos los medios y por los diferentes motivos de viaje.

El número promedio de viajes al día por habitante, de los datos de campo, es de 0.9, debajo de los promedios encontrados en ciudades similares en períodos conocidos como "normales". Vale indicar que en la fecha de la encuesta O/D las clases presenciales estaban suspendidas y como política se sugería el teletrabajo.

La movilidad se ha visto alterada drásticamente por la crisis de la COVID-19 y también en Santa Rosa. El confinamiento, el teletrabajo y las restricciones de movilidad han ejercido múltiples impactos en la movilidad diaria y el uso del transporte público se ha reducido de manera significativa, por lo que análisis posteriores deberán tomar en cuenta esta particularidad donde los patrones de movilidad se han visto alterados.

\section{Resultados.}

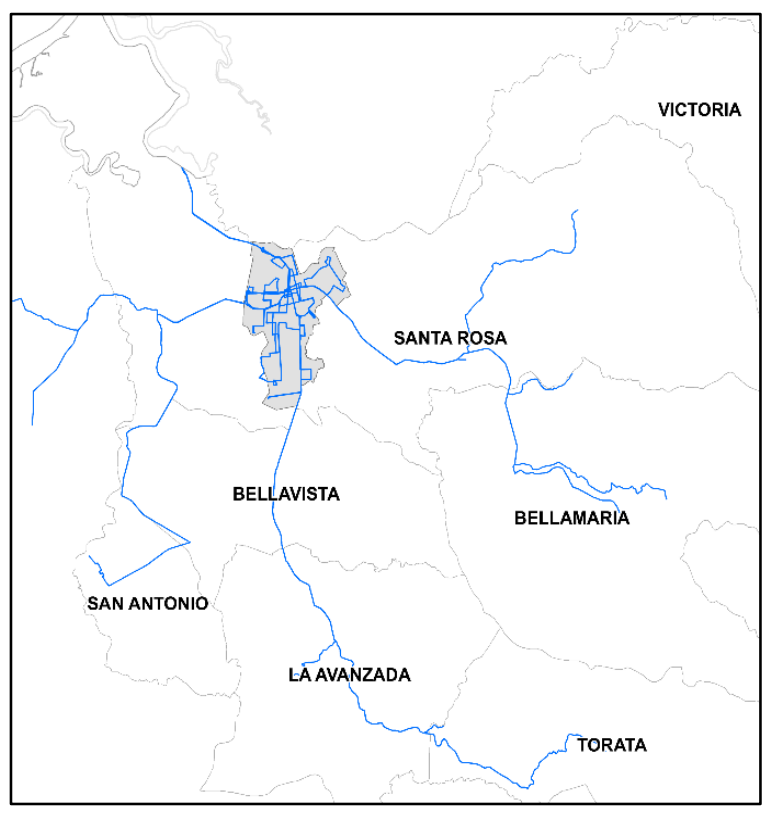

Los datos e información obtenida se refieren a la movilidad intracantonal, tanto en la parte urbana como rural, la información disponible es dispersa y obedece a los distintos fines para los que fueron contratados los diferentes estudios consultados, la gran mayoría de indicadores se calcularon con datos de fuente primaria, no existe un historial de validación de datos, por lo que se propone el seguimiento posterior.

Los Indicadores calculados se han clasificado en tres grandes grupos: Transporte público y comercial, tránsito-seguridad vial y vialidad.

en los que se enfatiza con principalmente: capacidad de flota de transporte público, la relación

Gráfico. 2. Transporte intracantonal y urbano.

Elaborado por el autor

de la flota de transporte comercial con la cantidad de habitantes, el empleo generado directamente, el Índice de Pasajeros Kilometro IPK, la cantidad de pasajeros transportados en transporte público y comercial, el recorrido promedio de buses y taxis, los gastos promedio que realiza la población en transporte, la cantidad de viajes según categorías, la distancia de los viajes en las diferentes modalidades, la partición modal, los motivos de viaje de la población, el tiempo de viaje en las distintas modalidades, la capacidad de viajes del transporte público, etc.

Respecto al tránsito se enfatizó en el Índice de motorización, viajes en medios motorizados, accidentabilidad anual en Santa Rosa, la tenencia de vehículos en hogares. En tanto que se 
proponen indicadores en vialidad respecto de la longitud de las vías, vías con prioridad para peatones y ciclistas, accesibilidad de los usuarios en las paradas de buses y velocidad de desplazamiento del transporte público.

En relación a la validación de los resultados vale precisar que la planeación del transporte en Latinoamérica tradicionalmente ha estado enfocada en el flujo de vehículos, en lugar de la circulación de personas o la accesibilidad. Si bien este paradigma ha cambiado, particularmente a nivel de política global debido al creciente enfoque en la movilidad sostenible, aún no ha llevado a un cambio de política en la subregión. Los planes de transporte de la mayoría de los países y muchas ciudades ya cuentan con referencias a la promoción del transporte sostenible, transporte activo y la mejora de las condiciones para el transporte público. (Moscoso, y otros 2020). Pero la evaluación sectorial con indicadores no es homogénea, pues la literatura internacional y regional se centra en la evaluación de los sistemas de transporte de las grandes ciudades y en Ecuador existe reciente referencia de evaluación e indicadores a partir del año 2012 con la publicación del MTOP "Herramientas de gestión para el transporte terrestre", posteriormente en 2014 el CNC publica una guía metodológica para planes de movilidad en el que de manera breve se menciona el tema indicadores.

El artículo "Identificación y análisis de indicadores de sostenibilidad para la movilidad" publicado en la revista de la Facultad de Arquitectura y Urbanismo de la Universidad de Cuenca el año 2017 constituye un referente importante, a pesar de referirse a una ciudad conocida como de tamaño medio y de enfocarse en la movilidad rural. Por lo tanto, la validación de los indicadores obtenidos para Santa Rosa se lo debe hacer con las reservas del caso ya que no existe una metodología específica, las existentes se centran en la movilidad de las grandes ciudades de Latinoamérica y en Ecuador los indicadores están dispersos tanto en su conformación como en su seguimiento en los Planes de Movilidad que realiza cada municipio. Al respecto la Asociación de Municipalidades del Ecuador, AME, realiza cada dos años la "Evaluación de la capacidad operativa en transporte, tránsito y seguridad vial" en la competencia asignada a los GADs pero este instrumento recoge información principalmente del cumplimiento de competencias asignadas.

Los indicadores susceptibles de validación sobre la base de la literatura existente son principalmente la tasa de motorización y que en Santa Rosa es relativamente alta, la tasa de accidentalidad en Santa Rosa es relativamente baja, la partición modal en Santa Rosa muestra una baja ocupación de el transporte público. El grueso de los indicadores deberá compararse con ciudades similares de hasta 100.000 habitantes.

Tabla 2. Indicadores de transporte.

\begin{tabular}{cccc}
\hline $\begin{array}{c}\text { Código } \\
\text { de } \\
\text { indicador }\end{array}$ & $\begin{array}{c}\text { Indicador de } \\
\text { Transporte }\end{array}$ & Resultado & Observaciones \\
\hline
\end{tabular}


Capacidad de flota de transporte público

TP2

Flota de transporte comercial por mil habitantes.

TP3 Empleo generado

TP4

dice de Pasajeros

Kilometro IPK

(pasajeros por $\mathrm{km}$ )

PVD

(pasajeros/vehículo/día)

en transporte público y

taxi

RMD

TP6

( $\mathrm{km} /$ vehículo/día) en transporte público.

Gasto promedio usuario de transporte público por motivo de estudios

Gasto promedio usuario

TP8 de transporte público por motivo trabajo

Gastos en transportación en

TP9 función del nivel de renta familiar disponible
Capacidad promedio en asientos operadora A: 48, operadora B: 39 y capacidad promedio de toda la flota: 45 .

Taxi 3,55; Carga Liviana 0,51; Transporte Escolar 0,40; Tricimotos 0,50; Transporte Público Intracantonal 0,63; Carga Pesada y Volquetes 1,42; y Carga Mixta 0,67.

Se estiman empleo directo generado por transporte público y comercial en 777 personas. La PEA de Santa Rosa es 30.998 personas, el $2.51 \%$ de la PEA está ocupada directamente en el transporte público y comercial.

El Índice de Pasajeros Kilómetro promedio en operación de buses en transporte intracantonal en de 5.1.

Promedio de pasajeros al día en transporte público: 827

Promedio de pasajeros transportados por viaje en taxi: 1.4

Promedio de kilómetros recorridos al día en transporte intracantonal: 161 Promedio de kilómetros recorridos al día en taxi: 255.

El promedio de gastos en transporte público por motivo de estudio es: 0.25 dólares.

Gasto promedio diario en transporte público intracantonal: 0.37 dólares. En transporte público intra e interprovincial es de 2.21 dólares.

Gasto promedio al día por hogar de 2.5 dólares y un total 69 dólares de gastos al mes por hogar.

El número de viajes en transporte público intracantonal al día es de 16.145, en
Mejorar la capacidad de los buses de transporte público, especialmente de los que hacen el servicio a la zona rural.

Debe incrementarse la cantidad de unidades de transporte público en relación a la población y limitar el número de unidades en el transporte de baja capacidad.

La actividad del transporte contribuye con un importante número de empleos directos.

Se considera un índice aceptable. Estos datos son de fuente secundaria y corresponde al año 2018, por lo que se debería levantar información actualizada. Puede mejorarse la cantidad promedio de pasajeros al día en transporte intracantonal y el promedio de pasajeros por viaje en taxi.

Se deberá actualizar la información.

Dato basado en encuestas de campo tomadas en octubre, sin clases presenciales y con nivel de movilidad reducida.

Se explica porque la tarifa en transporte público en Santa Rosa en área urbana es de 25 centavos y existe gran cantidad de viajes a lugares y cantones vecinos.

De las encuestas realizadas en Santa Rosa se obtiene un gasto promedio al día por hogar de 2.5 dólares y un total 69 dólares de gastos al mes por hogar.

Con política pública se deberá incrementar el 
ISSN: 2600-5859

Vol. 4, Nº1.1, p. 22-43, Febrero, 2021

transporte intra e interprovincial es de 8.253. Viajes totales estimados en Santa Rosa son 76.749 al día. En transporte intracantonal el porcentaje es de $21 \%$ y en transporte intra e interprovincial es de $11 \%$.

Trabajador cuenta propia $15 \%$,

TP11 de viajes por
ocupación-empleo.

TP12

$\%$ de viajes por escolaridad

TP13 Distancia de los viajes en transporte público intracantonal.

TP14

Distancia de los viajes en auto particular

Distancia de los viajes

TP15 a pie

TP16

Distancia de los viajes en bicicleta

TP17 Partición modal
Empleada(o) doméstica(o) 7\%, Empleado del Estado o de GADs 7\%, Empleado privado $11 \%$, Estudiante $7 \%$, Jornalero o peón $8 \%$, Otro $\quad 9 \%$.

Secundaria 33\%, Universitaria 21\%, Primaria $11 \%$.

Distancia promedio viajes en transporte público 2,6 km; 63,06\%

Distancia promedio viajes en transporte público 7,4 km; $36,57 \%$

Distancia promedio viajes en transporte público $12,3 \mathrm{~km} \quad 0,37 \%$ particular 4,7 km; 48,36\%; $13,4 \mathrm{~km}$; cabecera cantonal o

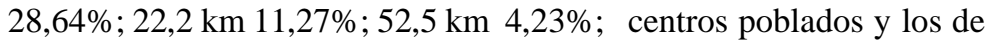
$30,9 \mathrm{~km} \mathrm{3,76 \%}$;

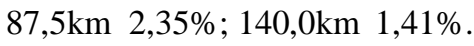

Distancia promedio viajes a pie en $\mathrm{km} 0,7$; 97,53\%.

Distancia promedio viajes a pie en $\mathrm{km} 1,9$ $2,47 \%$

Distancia promedio viajes en bicicleta en $\mathrm{km} \mathrm{2,2;83,08 \%}$

Distancia promedio viajes en bicicleta en km 6,3 16,92\%

Bus urbano 21,04\%, En vehículo propio $16,72 \%$, Bus Transporte intra o interprovincial 10,75\%, Motocicleta $9,89 \%$, Acompañante en vehículo propio $9,73 \%$, Taxi convencional 6,91\%, A pie $6,36 \%$, Bicicleta $5,10 \%$, Tricimoto $3,77 \%$, Taxi informal 3,53\%, Camión o volquete porcentaje de viajes en transporte público respecto de la partición modal.

La mayor cantidad de viajes son realizados por personas relacionadas con empleo particular y emprendimiento.

Los viajes realizados por personas con escolaridad de secundaria son representativos.

La cabecera cantonal del Santa Rosa tiene una extensión en línea recta de norte a sur de $5 \mathrm{~km} \mathrm{y}$ de oeste a este aproximadamente $\quad 2.5$ $\mathrm{km}$, por lo que los viajes en promedio de $2.6 \mathrm{~km}$ son mayoritarios.

Los viajes en promedio de $4.7 \mathrm{~km}$ son del $48 \%$ y están relacionados con la centros poblados y los de
mayor kilometraje con los viajes a sitios o cantones vecinos o resto del país.

Mayoritariamente $(97.53 \%)$ de los viajes se realizan con una distancia cerca al $1 \mathrm{~km}$ de longitud y mayores distancias tienen porcentajes menores. Se debe promover esta modalidad de desplazamiento.

Mayoritariamente

$(83.08 \%)$ de los viajes se realizan con una distancia alrededor de $2 \mathrm{~km}$ de longitud y mayores distancias tienen porcentajes menores.

En Santa Rosa, con información levantada a inicios de octubre del año 2020, vemos el bus urbano intracantonal tiene el $21.04 \%$. En auto particular y acompañante 
ISSN: 2600-5859

2,28\%, Camioneta doble cabina 1,33\%, Taxi Ejecutivo $1,18 \%$, Camioneta cabina simple $0,71 \%$, Camioneta informal $0,55 \%$, Escolar o institucional 0,16\%.

Trabajo 43,1\%, Compras $15,7 \%$, Trámites $10,8 \%$, Regreso a casa $8,9 \%$, Otros $7,9 \%$, Visita 7,8\%, Recreación esparcimiento 4,9\%, Estudios $0,8 \%$.

TP19

Tiempo de los viajes en transporte público

TP20

Tiempo de los viajes en auto particular

TP21

Tiempo de los viajes a pie

Tiempo de los viajes en bicicleta
Hasta 15 minutos $97,53 \%$, De 16 a 30 minutos $2,47 \%$.

Hasta 15 minutos63,06\%, De 16 a 30 minutos $36,57 \%$, De 31 a 45 minutos $0,37 \%$.

Hasta 15 minutos $48,36 \%$, De 16 a 30 minutos 28,64\%, De 31 a 45 minutos a 1 hora $3,76 \%$, De 2 a 3 horas 2,35\%, Más de 3 horas $1,41 \%$. 11,27\%, De 1 a 2 horas 4,23\%, De 46 min
Capacidad vehicular del transporte público colectivo por viajes y por habitantes. de auto particular se realizan el $26.45 \%$ de viajes en Santa Rosa.

El principal motivo de viaje en el Cantón Santa Rosa es el trabajo (43.1\%) seguido de las compras (15.7\%). Llama la atención el motivo de estudios con cerca del $1 \%$ cuando en condiciones "normales" este motivo supera el $10 \%$.

El tiempo de viaje en transporte público (buses) en su gran mayoría dura hasta 15 minutos con el $63 \%$, es decir los viajes son relativamente cortos en su mayoría.

En auto particular o como acompañante de vehículo particular se movilizan el $26.45 \%$ de la población en Santa Rosa. Los viajes en este medio mayoritariamente son de hasta 30 minutos $\mathrm{y}$ representan el $77 \%$.

Según la encuesta realizada, en el Cantón Santa Rosa se realizan estimativamente $\quad 4.880$ viajes a pie lo que representa el $6,36 \%$ del total de viajes; y de esta cantidad de viajes el $97.53 \%$ los hacen en viajes cortos de hasta 15 minutos.

Se realizan estimativamente $\quad 3.916$ viajes en bicicleta lo que representa el $5.10 \%$ del total de viajes; y de esta cantidad de viajes el $83.08 \%$ los hacen en viajes cortos de hasta 15 minutos.

El número total de viajes estimado según la encuesta deberá ser analizado con estudios posteriores, ya que arrojan totales inusuales, pues normalmente el 
ISSN: 2600-5859

TP24

Velocidad promedio de circulación transporte público.

Velocidad promedio de circulación de transporte público es $19 \mathrm{~km} / \mathrm{h}$.

Edad del parque

TP25 automotor del transporte público colectivo
Edad promedio del parque automotor de transporte público intracantonal: 14.9 años. promedio de viajes per cápita debe estar en alrededor de 1.5 y en Santa Rosa actualmente tenemos menos de un viaje per cápita.

Este indicador se obtuvo de el Estudio de Costos de operación realizado en el año 2018 en donde se determinó una velocidad promedio de recorrido de $19.4 \mathrm{~km} / \mathrm{h}$.

Se deberá gestionar para que la flota de transporte público intrantonal tenga un promedio de vida al menos de 8 años, lo que redundará en calidad de servicio y menos contaminación ambiental.

Elaborado por el autor.

Tabla 3. Indicadores de tránsito.

\begin{tabular}{cc}
\hline $\begin{array}{c}\text { Código } \\
\text { de } \\
\text { indicador }\end{array}$ & $\begin{array}{c}\text { Indicador de } \\
\text { Tránsito }\end{array}$ \\
\hline
\end{tabular}

TR1

Índice de motorización

TR2

Viajes en medios no motorizados

TR3
Accidentabilidad de tránsito anual
Vehículos por cada 1000 habitantes Ecuador (2018) 141,2, provincia de El Oro (2018) 166,1, cantón Santa Rosa (2015) $78,8,(2019) 121,5$.

El 6,4\% de los viajes se realizan a pie, el $5,1 \%$ se realizan en bicicleta y el $11,5 \%$ se realizan en medios no motorizados.

Siniestros por 100 mil habitantes (2019)

44,3; Lesionados por 100 mil habitantes 32,0 y Fallecidos por 100 mil habitantes 8,6 .

\section{Observaciones}

El índice de motorización es un indicador de la cantidad de vehículos respecto de la población. A mayor tasa, mayor tenencia de vehículos y los problemas consiguientes que son congestión y contaminación. La motorización en Santa Rosa pasa de 78.8 en 2015 a 121.5 en 2019, indicador de la acelerada motorización.

En el Cantón Santa Rosa se evidencia una baja cantidad de viajes en medios no motorizados.

El Cantón Santa Rosa tiene 8.6 fallecidos por cada 100 mil habitantes, al año 2019. Este índice si bien es relativamente bajo en comparación al de Ecuador y El Oro, no debe considerarse muy adecuado ya que normalmente se tiene un 
ISSN: 2600-5859

Vol. 4, Nº1.1, p. 22-43, Febrero, 2021

Tenencia de vehículos en hogares.
El 25\% de los hogares disponen de motocicletas El $21 \%$ de los hogares disponen de automóviles, el 8\% disponen de camionetas,.

Vehículos que

TR5 consumen energía alternativa 0 renovable
No existen registrados en el cantón vehículos que utilicen energías renovables. subregistro y se

contabilizan solamente los que fallecen en sitio y no los que fallecen posteriormente.

De acuerdo a la encuesta levantada, el $25 \%$ de los hogares dispone de motocicleta, el $21 \%$ de automóvil y el $8 \%$ de camioneta; el $60 \%$ de los hogares tiene algún tipo de vehículo.

Promover el uso de vehículos con energías alternativas.

Elaborado por el autor.

Tabla 4. Indicadores de vialidad

\begin{tabular}{|c|c|c|c|}
\hline $\begin{array}{c}\text { Código } \\
\text { de } \\
\text { indicador }\end{array}$ & $\begin{array}{l}\text { Indicador de } \\
\text { vialidad }\end{array}$ & Resultado & Observaciones \\
\hline V1 & Km de vías. & $\begin{array}{l}\text { Hormigón 80,98 km 21\%; Asfalto } \\
\text { 80,12km 17\%; Lastre } 104,33 \mathrm{~km} \mathrm{27 \% ;} \\
\text { Tierra 113,44 km 35\%. }\end{array}$ & $\begin{array}{l}\text { En la vialidad cantonal } \\
\text { vemos que el } 38 \% \text { son de } \\
\text { hormigón o asfalto y el } \\
\text { restante porcentaje son de } \\
\text { lastre o tierra. }\end{array}$ \\
\hline V2 & $\begin{array}{l}\text { Intersecciones } \\
\text { semaforizadas }\end{array}$ & Existen 36 intersecciones semaforizadas. & $\begin{array}{l}\text { Esto indica la presencia de } \\
\text { un importante flujo } \\
\text { vehicular y de conflictos } \\
\text { que han hecho necesario } \\
\text { su implementación. }\end{array}$ \\
\hline V3 & $\begin{array}{l}\text { Vías con prioridad } \\
\text { para peatones }\end{array}$ & $\begin{array}{l}\text { De } 161 \mathrm{~km} \text { de vías de hormigón o asfalto, } \\
12 \text { son destinadas vías peatonales, que } \\
\text { representa el } 7 \% \text {. }\end{array}$ & $\begin{array}{l}\text { El porcentaje de vías con } \\
\text { prioridad para peatones se } \\
\text { deberá incrementar. }\end{array}$ \\
\hline V4 & $\begin{array}{l}\text { Vías con prioridad } \\
\text { para ciclistas }\end{array}$ & $\begin{array}{l}\text { De los } 161 \mathrm{~km} \text { de vías de hormigón o } \\
\text { asfalto, } 10 \text { son destinadas ciclovías, que } \\
\text { representa el } 6 \% \text {. }\end{array}$ & $\begin{array}{l}\text { El porcentaje de vías con } \\
\text { prioridad para ciclistas se } \\
\text { deberá incrementar. }\end{array}$ \\
\hline V5 & $\begin{array}{l}\text { Acceso a personas } \\
\text { con movilidad } \\
\text { especial en la parada }\end{array}$ & $\begin{array}{l}\text { En Cantón Santa Rosa existen } 564 \text { paras o } \\
\text { paraderos de buses para el sistema de } \\
\text { transporte intracantonal, en } 22 \text { de ellas se } \\
\text { han implementado apoyos para personas } \\
\text { con discapacidad y } 18 \text { cuentan con rampas } \\
\text { de acceso. }\end{array}$ & $\begin{array}{l}\text { Se debe mejorar los } \\
\text { accesorios de las paradas } \\
\text { que permitan la } \\
\text { accesibilidad adecuada de } \\
\text { personas con } \\
\text { discapacidad. }\end{array}$ \\
\hline V6 & $\begin{array}{l}\text { Puntos seguros de } \\
\text { paradas }\end{array}$ & $\begin{array}{l}\text { En el Cantón Santa Rosa existen } 564 \\
\text { paradas o paraderos para el transporte } \\
\text { intracantonal, en } 18 \text { de ellas se cuenta con } \\
\text { caseta o infraestructura. }\end{array}$ & $\begin{array}{l}\text { Se debe mejorar la } \\
\text { infraestructura de las } \\
\text { paradas que permitan la } \\
\text { accesibilidad adecuada de } \\
\text { personas r con } \\
\text { discapacidad. }\end{array}$ \\
\hline V7 & $\begin{array}{l}\text { Velocidad de } \\
\text { desplazamiento } \\
\text { media del transporte } \\
\text { público. }\end{array}$ & $\begin{array}{l}\text { La velocidad promedio en transporte } \\
\text { público intracantonal es de } 19.4 \mathrm{~km} 7 \mathrm{~h} \text {, en } \\
\text { tanto que la velocidad promedio en } \\
\text { transporte particular es de } 35 \mathrm{~km} / \mathrm{h} \text {. }\end{array}$ & $\begin{array}{l}\text { Se considera una } \\
\text { velocidad promedio } \\
\text { adecuada. }\end{array}$ \\
\hline
\end{tabular}




\begin{tabular}{llll}
\hline Km del sistema del & $\begin{array}{l}\text { 100,8 Km por cada 100 mil hab de rutas de } \\
\text { transporte urbano. } \\
280.2 \mathrm{Km} \text { por cada 100 mil hab rutas } \\
\text { transporte rural. }\end{array}$ & $\begin{array}{l}\text { Se deberá incrementar el } \\
\text { número de km de vías de } \\
\text { transporte público y } \\
\text { especialmente mejorar sus } \\
\text { transporte por } \\
100,000 \text { habitantes } \\
\text { relacionadicas } \\
\text { prioridad. }\end{array}$ \\
\hline
\end{tabular}

Elaborado por el autor.

\section{Conclusiones}

- La importancia de contar con indicadores de movilidad sostenible radica en el hecho que el sector puede ser evaluado consecutivamente y verificar los avances o retrocesos en cada uno de los aspectos sujetos de medición, por lo que es deseable que se plantee como política de las autoridades respectivas hacer seguimiento de los indicadores y ampliar la base de los mismos, incorporando temas sociales, económicos y ambientales. Los niveles de movilidad, producto de las restricciones de la emergencia sanitaria, se han contraído significativamente en comparación con datos levantados en años anteriores y en cantones similares, por lo que el análisis deberá hacer reparo en esta circunstancia.

- Los datos obtenidos revelan una alta proporción de viajes realizados en medios de transporte individual y particular, predomina el uso importante del auto particular y de las modalidades de transporte comercial de baja capacidad, registrándose un incremento de la tasa de motorización comparando los años 2015 y 2019.

- Los indicadores de operación del transporte público pueden mejorarse mediante la implementación de políticas públicas que prioricen el transporte público colectivo sobre el particular e individual, con la finalidad de hacer mejor uso del espacio vial y público disponible y mejorar el medio ambiente que se ve afectado por el uso intensivo del auto particular. La partición modal prevaleciente en la actualidad no favorece la movilidad sostenible ya que en el sistema de transporte público intracantonal apenas se realizan el $21.04 \%$ de los viajes, los viajes a pie son el $6.36 \%$ y los viajes en bicicleta el 5.10\%, tomando en cuenta que Santa Rosa por su configuración topográfica y amplitud de vías ofrece la oportunidad para desarrollar modos de transporte no motorizados.

- El 38\% de la vialidad cantonal está compuesta por vías de hormigón o asfalto y el restante porcentaje son de lastre o tierra, el porcentaje de vías con prioridad para peatones es de $7 \%$ y el porcentaje de vías con prioridad para ciclistas es del $6 \%$, circunstancia absolutamente susceptible de mejora. La infraestructura de paradas de bus y facilidades para personas de grupos vulnerables es deficitaria en el sistema de transporte público en el Cantón Santa Rosa. 


\section{Referencias Bibliográficos}

Álvarez Valencia, Carolina . «La movilidad sostenible como política global y su consolidación hacia el futuro en la ciudad de Medellín en respuesta a la ocupación territorial y sus problemáticas.» Medellín, 2016.

CAF. «Desarrollo urbano y movilidad en América Latina. .» 2011.

Consejo Nacional de Competencias. «Resolución 006-CNC-2012.» 2012.

Consejo Nacional de Competencias. «Guía metodológica para la formulación de planes de movilidad para los gobiernos autónomos descentralizados municipales del modelo de gestión C.» Quito, 2014.

Díaz Muñoz, María Ángeles, Carolina Cantergiani, María Jesús Salado García, Carolina Rojas Quezada, y Martinez S Gutiérrez. «Propuesta de un sistema de indicadores de sostenibilidad para la movilidad y el transporte urbanos. Aplicación mediante Sig a la ciudad de Alcalá de Henares.» 2007: 1916.

Eguiguren Meneses , Francisco Javier. «¿Caminando A La Movilidad Urbana Sostenible?: Desafíos De La Implementación De Políticas En La Ciudad De Quito.» Quito: Flacso, Mayo de 2015.

Flores Juca, Enrique, Justo García Navarro, Jessica Chica Carmona, y Estefania Mora Arias. «Identificación y análisis de indicadores de sostenibilidad para la movilidad.» Estoa. Revista de la Facultad de Arquitectura y Urbanismo de la Universidad de Cuenca , 2017.

GAD Municipal Del Cantón Santa Rosa. «Actualización Del Plan De Desarrollo Y Ordenamiento Territorial 2019-2023 Y Elaboración Delplan De Uso Y Gestión Del Suelo 2020-2032 Del Cantón Santa Rosa - Provincia De El Oro.» Santa Rosa, 2020.

García-Schilardi, María Emilia. «Transporte público colectivo: Su rol en los procesos de inclusión social.» 2013: 42.

Holden, Erling. Achieving Sustainable Mobility. Everyday and Leisure-time Travel in the EU. Burlington: Ashgate, 2007.

Instituto para la Diversificación y Ahorro, IDAE. «PMUS: Guía práctica para la elaboración e implantación de planes de movilidad sostenible.»IDAE, 2006: 16.

Ministerio de Transporte y Obras Públicas. «Herramientas de gestión para el transporte terrestre.» Quito. p 224, 2012.

Moscoso, Marina, Thomas van Laake, Lina Marcela Quiñones, Carlos Felipe Pardo, y Darío Hidalgo. Transporte urbano sostenible en América Latina: evaluaciones y recomendaciones para politicas de movilidad. Bogotá.: Despacio, 2020.

Motos Cascales, Gloria. «Análsisi de indicadores de movilidad urbana sostenible.» Universidad Politécnica de Cartagena. Escuela Técnica Superior de Ingeniería de Caminos, Canales y Puertos y de Ingeniería de Minas., 2019.

Ortiz Álvarez, María Inés, María Elena Cea Herrera, y Jorge González Sánchez. «Escenarios demográficos en América Latina y el Caribe.» Red de Revistas Científicas de América Latina, el Caribe, España y Portugal, 2003. 
ISSN: 2600-5859

www.concienciadigital.org

Vol. 4, N¹.1, p. 22-43, Febrero, 2021

Pozueta, Julio. «Movilidad Y Planeamiento Sostenible: Hacia una consideración inteligente Hacia una consideración inteligente del transporte y la movilidad en el planeamiento y en el diseño urbano.» Cuadernos De Investigación Urbanística, 2000: 109 pag 46.

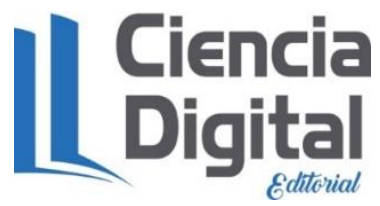




\section{PARA CITAR EL ARTÍCULO INDEXADO.}

Carpio Arévalo, J. A. (2021). La aplicabilidad de la normativa jurídica ecuatoriana, en torno a los PDOT cantonales. Estudio de caso del GAD Municipal del cantón Cuenca. ConcienciaDigital, 4(1.1), 6-21. https://doi.org/10.33262/concienciadigital.v4i1.1.1544

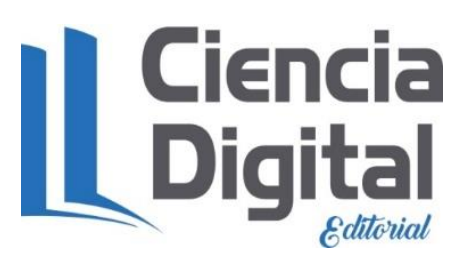

El artículo que se publica es de exclusiva responsabilidad de los autores y no necesariamente reflejan el pensamiento de la Revista Conciencia Digital.

El artículo queda en propiedad de la revista y, por tanto, su publicación parcial y/o total en otro medio tiene que ser autorizado por el director de la Revista Conciencia Digital.
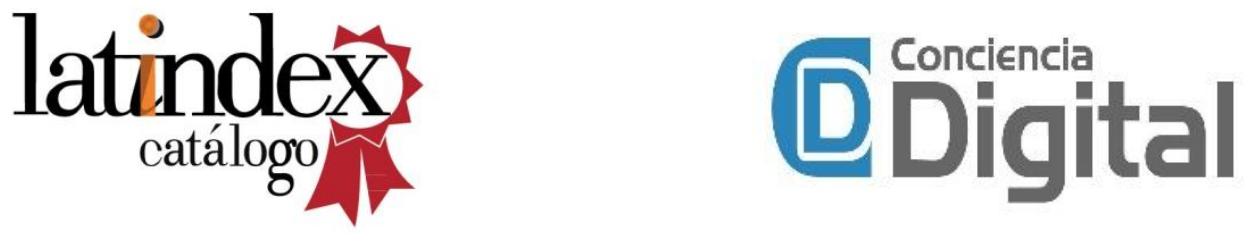